\title{
Intergenerational transmission of child abuse: Predictors of child abuse potential among racially diverse women residing in domestic violence shelters
}

\author{
RaeAnn E. Anderson ${ }^{a, c}$, Larissa-Jayne Edwards ${ }^{b}$, Kristin E. Silver ${ }^{b}$, and Dawn M. Johnson ${ }^{b}$ \\ aKent State University, Psychological Sciences, 144 Kent Hall, Kent, OH 44242 \\ bUniversity of Akron, Department of Psychology, Akron, OH 44325-4301 \\ cUniversity of North Dakota, Psychology, 2000 Columbia Hall, Grand Forks, ND 58202
}

\begin{abstract}
Parental risk for perpetrating child abuse is frequently associated with intergenerational patterns of abuse: being abused increases the risk for future abuse. Yet, the mechanisms of intergenerational abuse are unclear, and the risk factors for perpetrating child abuse are interrelated. Research suggests that history of childhood abuse, psychiatric distress, and exposure to intimate partner violence (IPV) are all related risk factors for perpetrating child abuse. We investigated these three risk factors using the developmental psychopathology framework in a racially diverse sample of high-risk women: women residing in domestic violence shelters. 211 mothers residing in domestic violence shelters completed measures of their own childhood abuse (defined narrowly in a 10-item self-report survey), exposure to and severity of IPV victimization, and structured interviews to diagnose psychiatric disorders. We utilized a hierarchical regression model to predict child abuse potential, accounting for risk factors in blocks roughly representing theorized temporal relationships: childhood abuse followed by psychiatric diagnoses, and then recency of exposure to IPV. Consistent with hypotheses, the strongest predictor of current child abuse potential was the psychiatric diagnosis of PTSD. Mediation tests further explicated that the relationship between maternal history of childhood sexual abuse and current potential for perpetrating child abuse is mediated by IPV-related PTSD symptoms. Results suggest that IPV-related PTSD symptoms, rather than exposure to abuse (i.e., childhood abuse or IPV), is most strongly associated with child abuse potential in recent IPV survivors. Interventions which can ameliorate maternal psychopathology and provide resources are recommended for these vulnerable families.
\end{abstract}

\section{Keywords}

child abuse potential; child abuse; intimate partner violence; PTSD; borderline personality

Correspondence to: RaeAnn E. Anderson.

Publisher's Disclaimer: This is a PDF file of an unedited manuscript that has been accepted for publication. As a service to our customers we are providing this early version of the manuscript. The manuscript will undergo copyediting, typesetting, and review of the resulting proof before it is published in its final citable form. Please note that during the production process errors may be discovered which could affect the content, and all legal disclaimers that apply to the journal pertain. 
Childhood physical and sexual abuse, defined as any act(s) on the part of a caretaker which result in harm, are relatively common and harmful experiences for many children in the US (USDHHS, 2012). In 2011, there were 686,000 confirmed victims of childhood abuse or neglect, of which 17.6 percent were victims of physical abuse and $9 \%$ of sexual abuse (USDHHS, 2012). Actual numbers are much higher due to the systemic under-reporting of these crimes (Juby, Downs, \& Rindel, 2014). The consequences of childhood abuse are many and long-lasting; people with a history of childhood abuse are more likely to experience emotional distress in the forms of depression and anxiety (Juby et al., 2014). In addition, much evidence links experiences of childhood abuse to adult experiences of abuse, such as intimate partner violence victimization (IPV: Hamby \& Grych, 2013; Widom \& Wilson, 2015). In recent years, both scholars of childhood abuse and IPV have begun investigating these interrelationships. In particular, researchers have studied the links between parental histories of childhood abuse, adult experiences of IPV, and how these experiences may then transmit risk for child abuse to the next generation (e.g., Noll, Trickett, Harris \& Putnam, 2008).

One manner of examining this pathway has been the construct of child abuse potential (CAP). CAP is a construct designed to assess a range of established risk factors (e.g., parenting distress, rigidity, family conflict) for perpetrating moderate to severe physical child abuse (e.g., hitting or pushing a child) in parents that can be assessed without triggering a socially desirable response style in participants. CAP is a multidimensional construct meant to represent overall risk for engaging in physical child abuse that can occur via a variety of pathways (Milner \& Wimberley, 1979). The development of the Child Abuse Potential Inventory and the Brief Child Abuse Potential Inventory included criterion validity research designs with cases referred to child protective services (Milner \& Wimberly, 1979; Ondersma, Chaffin, Mullins, \& LeBreton, 2005); thus, it is considered a highly valid, although not necessarily face-valid, measure of CAP (for a critical review, see Walker \& Davies, 2010). The goal of the current study was to investigate a comprehensive model of risk factors for child abuse potential using a developmental psychopathology framework in a high-risk group: mothers in domestic violence shelters. We also sought to provide descriptive data on child abuse potential scores and explore dimensions of child abuse potential in this unique sample.

\section{History of Childhood Abuse, Maternal Psychopathology, and Later Child Abuse Potential}

Many studies have examined the relationships between maternal history of childhood abuse and CAP (e.g., Jaffe, Cranston, \& Shadlow, 2012, childhood physical abuse; Bert et al., 2009; childhood physical abuse and neglect). Some have found that indicators of psychological health, such as maternal responsivity, parenting, or trauma symptoms (e.g., Milner et al., 2010), mediate these relationships. This suggests that it is not the presence of childhood abuse per se, but the context of the abuse and the ongoing consequences of these experiences that increase child abuse potential. Specifically, constructs such as distress related to childhood abuse, frequency of abuse, and relationship to the perpetrator are linked to differential clinical outcomes in some research (Hazen, Connelly, Roesch, Hough \& 
Landsverk, 2009; Charak, DiLillo, Messman-Moore, \& Gratz, 2017). Thus, more comprehensively assessing the characteristics and context of childhood abuse is important for further explicating and understanding the ongoing consequences of childhood abuse and may explain mixed findings in the literature (Haselschwedt, Savasuk-Luxton, \& Hlavaty, 2017).

Psychopathology is a common consequence of childhood abuse, as well as an independent risk factor for child abuse potential. Psychiatric symptoms interfere with accurate attributions, impair judgment, and worsen functioning and emotional regulation, ultimately impairing the sufferer's ability to parent effectively (Rodriguez, Smith \& Silvia, 2016; BergNielsen, Vikan, \& Dahl, 2002). Yet, it is important to investigate the effects of specific types of psychopathology, as some symptoms may be more debilitating than others and require different intervention strategies. For example, emotional numbing symptoms such as those seen in depression and posttraumatic stress disorder (PTSD) may be related to less maternal empathy or responsivity (Bert et al., 2009). In contrast, the emotional reactivity associated with hyperarousal (in PTSD) and borderline personality disorder (BPD) is associated with greater negative attributions about children's behavior, which can increase child abuse potential (Rodriguez, Smith, \& Silvia, 2016). Depression, BPD, and substance use are linked to an increased risk of parenting stress and child abuse (Kelley, Lawrence, Milletich, Hollis, \& Henson, 2015; Laulik, Chou, Browne, \& Allam, 2013; Shenk et al., 2017). To wit, 56\% of mothers in opioid-substitution therapy scored in the high-risk range for child abuse potential (Dawe, Taplin, \& Mattick, 2017). Yet, in many samples, including among women in shelter, psychiatric comorbidity is the norm, with the most common diagnoses including PTSD, depression, substance use disorders, and BPD (Johnson, Zlotnick, \& Perez, 2008). Research is needed that assesses multiple, specific diagnoses, as outpatient treatment is often predicated on specific diagnoses rather than level of distress.

\section{Maternal Psychopathology, IPV, and Child Abuse Potential}

Research demonstrates that psychopathology increases risk for experiencing IPV victimization (Iverson, Litwack, Pineles, Vaughn, \& Resick, 2013; Johnson et al., 2013), and IPV itself is another risk factor for child abuse (Rodriguez, 2006). Thus, psychopathology may play a central role in understanding child abuse potential among survivors of severe trauma. As traumas accumulate, so do psychiatric symptoms and impairment, which further increases one's vulnerability to violence and abuse (Myers et al., 2015; Hamby \& Grych, 2013). However, research on the relationships of CAP < mi $>$ IPV and IPV <mi> psychopathology is often silo-ed in separate literatures (Banyard, 2013; MacMillan \& Wathen, 2005). For example, Whiting and colleagues (2009) found adult psychiatric diagnoses (PTSD, alcohol dependence) increased the likelihood of adult IPV victimization in an epidemiological sample of childhood abuse survivors. Smith and colleagues (2014) found that negative emotion mediated the relationship between child abuse and CAP in a sample of low-income, racially diverse mothers. Although Whiting et al. (2009) used a similar strategy to Smith et al. (2014) to assess child abuse history, Whiting et al. (2009) did not assess current child abuse potential. Smith et al. (2009) examined psychiatric distress broadly, rather than utilizing specific diagnoses. 


\section{Theories of Intergenerational Transmission of Childhood Abuse and Women in Shelter}

Research and theory are beginning to integrate how and why people with a history of childhood abuse are at greater risk for IPV victimization as adults (childhood abuse $<\mathrm{mi}>$ IPV) and at greater risk perpetrating abuse towards their children (childhood abuse $<\mathrm{mi}>$ CAP). Historically, the predominant theoretical model was social learning theory, which posits that having experienced childhood abuse normalizes the experience and thus facilitates IPV and child abuse potential via changes in attitudes and behavior regarding violence (Widom \& Wilson, 2014). However, this theory poorly accounts for the role of psychopathology. The developmental psychopathology perspective emphasizes how psychopathology, as a result of childhood abuse, increases risk for IPV and child abuse potential by altering developmental trajectories and downstream processes such as emotion regulation and parenting behavior. This perspective is promising because there are reliable, effective treatment options for many forms of psychopathology, offering hope that the effective use of existing resources can reduce psychological suffering along with the risk of child abuse. Although a cross-sectional study does not allow an examination of developmental trajectories, it allows multiple levels of analysis within the same person, a recommendation of this theory (Cicchetti \& Toth, 2009).

Racially diverse mothers in domestic violence shelters are an important and under-studied group for utilizing the developmental psychopathology framework to understand the intergenerational transmission of abuse. Their risk for engaging in child abuse is likely complex and their histories of abuse and associated psychopathology severe (Johnson et al., 2008; Gobin et al., 2013). Women in shelter are struggling to overcome individual and systemic barriers that may reduce their risk of child abuse potential by reducing their exposure to IPV. Indeed, Rodriguez (2006) found that women post-transitional housing had lower CAP scores than women in shelter. However, women in shelter are also at increased risk for CAP due to ongoing challenges in their lives, such as housing instability and psychiatric distress. Few studies have examined CAP in this high-risk sample from a developmental psychopathology approach. To our knowledge, Rodriguez (2006) is the only published research examining CAP in women in shelter. This study found that women in shelter had higher CAP scores on average than adjudicated abusers; results also showed a strong relationship between psychiatric distress and CAP (Rodriguez, 2006).

\section{Current Study and Hypotheses}

Women in domestic violence shelters are a marginalized population experiencing a confluence of risk factors while seeking safety and stability. The current study aimed to address limitations in previous research by testing a more complex model of child abuse potential using a developmental psychopathology framework in a unique and highly relevant sample: racially diverse women in domestic violence shelters. Descriptive data is needed to contextualize CAP scores in this population, and exploratory research is needed to understand the how the subscales of the Brief Child Abuse Potential Inventory (BCAP) may be related to psychopathology in unique, high-risk populations. It is especially important to 
utilize accurate screening and diagnostic tools for child abuse potential in a setting where families are attempting to build resilience and empowerment while struggling to meet basic needs. Indeed, challenges in parenting are a serious stressor for mothers during and after their time in the shelter (Ham-Rowbottom, Gordon, Jarvis \& Novaco, 2005). To our understanding, only one study has explored the BCAP subscales in a high-risk population: opioid-dependent mothers (Dawe et al., 2017). No prior studies have explored the relationship between BCAPI subscales and psychopathology.

Aim 1: Test a comprehensive, developmental psychopathology model of child abuse potential using the developmental psychopathology framework. We focused on three diagnoses very common in women in shelter (depression, PTSD, and borderline personality disorder; Johnson, Zlotnick, \& Perez, 2008), all of which are strongly associated with history of childhood abuse (Ackerman, Newton, McPherson, Jones \& Dykman, 1998; Filipas \& Ullman, 2006; Rogosch \& Cicchetti, 2005). We utilized multiple IPV variables, such as number of days since contact with the abuser, rather than solely severity of IPV, to counter possible ceiling effects, as all study participants had experienced IPV severe enough to necessitate seeking shelter. We assessed only moderate-severe maternal histories of childhood physical and sexual abuse as well as variables capturing the context this childhood abuse, such as distress and relationship to the perpetrator (Haselschwerdt et la., 2017). Hypotheses: IPV variables and current psychopathology (especially trauma-related psychopathology: PTSD and BPD), rather than maternal history of childhood victimization, will be predictive of child abuse potential scores, in line with the proximal occurrence of these variables and consistent with the findings of Smith et al. (2014).

Aim 2: Informed by the results of Aim 1, we will test specific mediation models assessing the relationship between maternal history of childhood abuse variables, IPV-related PTSD, and current child abuse potential.

Aim 3: Provide descriptive data on BCAP total scores for this high-risk, unique population. No prior research has published BCAP scores in this population. Hypothesis: The percentage of the sample with a high-risk score will be higher in this population compared to other published research, consistent with the findings of Rodriguez (2006).

Aim 4: Investigate potential differences in BCAP subscale scores by diagnosis, given the utility of specific diagnoses in understanding CAP and as intervention targets. Ondersma et al. (2005) highly recommends research on the subscales to investigate their potential use in understanding CAP. Yet, little research has explored the BCAP subscales, and no studies have gathered data on the BCAP subscales with samples of women in domestic violence shelters. Given the paucity of research, we consider this aim exploratory.

\section{Methods}

\section{Participants}

Participants were 273 women aged 18 and older residing in one of six domestic violence shelters in Northeast Ohio. Participants were recruited as part of a larger study examining PTSD treatment in domestic violence shelters. Analyses for this paper focus on the sub- 
sample of women with children $(n=250)$ who completed a valid measure of child abuse potential ( $n=211$, see also Data Cleaning). Thus, our final sample of 211 women ranged in age from 19 to $59(M=35.2, S D=8.8)$; most had less than a college education $(62.0 \%)$, were unemployed $(85.8 \%)$, low income $(89.6 \%$ earned $<\$ 10,000)$, and receiving government aid (80.6\%). The sample was racially diverse: White (46.4\%), Black/African American (44.1\%), multiracial (8.5\%), and Native American (0.5\%). Ethnically, 5.2\% identified as Latina. Most had never been married (50.7\%), and many were divorced (22.7\%), or separated (15.2\%). The majority of participants identified themselves as heterosexual (92.9\%), fewer as bisexual (6.6\%), and very few as lesbian $(0.5 \%)$.

\section{Materials}

Childhood abuse history.-History of physical and sexual abuse before age 18 was assessed using the Traumatic Stress Schedule (TSS: Norris \& Perilla, 1996). For this study, we modified the instructions to ensure that participants did not report traumas related to the IPV that brought them to shelter. The TSS uses ten items to assess trauma history; each item describes a traumatic event, the frequency of the event, the extent of distress experienced during the event, and the victim's relationship to the perpetrator (e.g., spouse/partner, friend, family member, stranger, other). Follow-up items were added to the TSS to assess age at the time of the trauma to ensure peer victimization, family abuse, and IPV were separately identified.

The TSS item "did anyone ever beat you up or attack you?" was used to assess history of childhood physical abuse. This is consistent with the BCAP's focus on moderate to severe physical abuse. The TSS item "did anyone ever make you have sex by using force or threatening to harm you? This includes any type of unwanted sexual activity," was used to assess childhood sexual abuse. Participants rated their distress related to these traumas, "to what extent were you distressed by the event?" on a scale from 1-'not at all' to 7-'extremely'. Items were coded positively for history of childhood physical or sexual abuse if participants reported that they were 17 or younger at the time of the event. The TSS has good validity; participants who endorse TSS items are more likely to experience PTSD symptoms (Norris, 1992). The TSS also has good test-retest reliability, with strong one-week test-retest correlations (Norris \& Perilla, 1992). The TSS has previously been used to assess childhood abuse history (Smith, Level, \& Chamberlain, 2006; Schneider, Baumrind \& Kimerling, 2007). The TSS childhood abuse items are similar to the Adverse Childhood Experiences (ACEs) items (Felitti et al., 1998). For example, the ACES childhood physical abuse item reads, "did a parent or other adult in the household often push, grab, slap, or throw something at you?" While it is likely that the TSS under-identifies less severe cases of childhood abuse, it is highly unlikely to identify false positives, making it appropriate for this sample of women with extreme abuse histories.

Major Depression and Substance Use Disorders.-The mood disorders and substance use disorders modules from the Structured Clinical Interview for DSM-IV-I/P (SCID: First, Spitzer, Gibbon, \& Williams, 2002) were used to assess major depression and substance use disorders (SUDs). For analyses, alcohol and drug use diagnoses were combined under the umbrella of SUDs. Current MDD and SUD diagnoses were used for 
analyses. Inter-rater reliability in this study was examined for 13 randomly selected interviews for MDD diagnoses; reliability was excellent $(\kappa=1.0)$.

The Center for Epidemiologic Studies Depression Scale-Revised (CESD-R: Eaton, Smith, Ybarra, Muntaner, \& Tien, 2004) was used to assess the severity of depression symptoms for those who met criteria for current depression based on the SCID. In the present study, participants were asked to rate how often they had experienced each symptom in the past week (rather than the past month) on a scale ranging from 0 ('Not at all or less than one day') to 4 ('Nearly every day for the past week'). In the present study, Cronbach's alpha was 0.91 .

Borderline Personality Disorder.-To assess BPD, the BPD module of the Structured Clinical Interview for DSM-IV Personality Disorders Interview was administered; this measure has excellent reliability and validity (First et al., 2002; Lobbestael, Leurgan \& Arntz, 2011).

PTSD.-The Clinician-Administered PTSD Scale (CAPS: Blake et al., 1995) is a structured interview designated the gold standard for PTSD assessment. The CAPS demonstrates good reliability and validity (Weiss, 2004). The CAPS was used to assess the presence of IPVrelated PTSD diagnoses and the severity of PTSD symptoms. The CAPS assesses the frequency and intensity of each DSM-IV-TR symptom of PTSD as well as symptoms associated with severity of PTSD (e.g., dissociation). All CAPS items were used to calculate past month PTSD severity scores for those who met criteria for PTSD related to IPV. The CAPS demonstrated strong reliability, Cronbach's alpha $=0.93$. Inter-rater reliability for 20 randomly selected interviews for PTSD diagnoses was excellent $(\kappa=1.0)$.

\section{The Severity of Violence Against Women Scales (SVAWS: Marshall, 1992).-}

The SVAWS uses 46 items to assess the frequency of physical and sexual aggression from a partner in the past month. All items are rated on a 5-point scale from 1 - "never" to 4 "many times" and including an option "it did not happen in the month prior but did before." An example item is: "how often did he throw an object at you?" For this study, items were modified to be gender-neutral. The SVAWS has been widely used and shows good reliability in a variety of populations (Trimble, Nava \& McFarlane, 2013; Lannert, Levendosky \& Bogat, 2013). Internal consistency reliability was excellent in this sample, $a=.97$. Scores related to violence experienced from the partner which necessitated their seeking shelter were used in this study. Follow-up questions were presented after the SVAWS to assess contact with the abuser since entering shelter. This data was used to calculate the number of days since contact with the abuser.

The Psychological Maltreatment of Women Inventory (PMWI: Tolman, 1999).This study used a shortened version (14 items) of the PMWI. The directions were altered to specifically assess behavior the woman may have experienced in her relationship with the partner who caused her to enter shelter in the past month. The PMWI is designed to measure dominance, isolation, verbal abuse, and emotional abuse; a total score is then calculated. PMWI scores are significantly associated with measures of physical abuse and treatment seeking, indicating validity (Tolman, 1999). Items are rated on a 5-point frequency scale 
ranging from 1 - "never" to 5 - "very frequently." Internal consistency was good in this sample, $a=.90$.

Primary Outcome: The Brief Child Abuse Potential Inventory (BCAP).-The BCAP is a shortened version of the 133 item Child Abuse Potential Inventory (Ondersma et al., 2005). All 33 BCAP items are presented in a dichotomous "agree" or "disagree" format. The BCAP items can be reduced to two types of subscales: an overall abuse risk subscale (24 items) and a validity scale ( 9 items). The validity scale has two sub-components: a random responding subscale ( 3 items) and a lie scale (6 items). One item from the Lie subscale was accidentally omitted during data collection, resulting in 5 lie scale items being administered.

The abuse risk scale items include prompts such as, "I am a happy person" and "My family has problems getting along" that individually do not necessarily reflect abuse risk but cumulatively indicate familial conflict and instability. The random responding scale includes items that would be very uncommon for a parent to respond "no" to, such as "children should not learn how to swim." The lie scale contains items that might trigger socially desirable responding such as, "I sometimes say bad words." Kudar-Richardson-20 internal consistency for the abuse risk scale was .76. There are seven subscales designed for research, Kudar-Richardson-20 internal consistency, which are listed in parentheses: distress (.81), family conflict (.51), rigidity (.83), happiness (.19), persecution (.47), loneliness (.85), and financial stress (.39). For Aim 3, we only examined subscales with internal consistency scores above .70 .

\section{Procedures}

Data were collected from 2013 - 2015 in six domestic violence shelters in Northeast Ohio under the supervision of the Institutional Review Board of the final author. The study was advertised at each shelter through brochures and posted signs in shelter, as well as recruitment sessions during shelter meetings. Interested participants were asked to call a confidential research line where the participants were provided more information on the study and screened to confirm that the abuse bringing women to shelter was from an intimate partner. If interested and eligible, participants were scheduled for an interview in shelter. The diagnostic interview was completed first, followed by self-report questionnaires. Self-report questionnaires were administered in the following set order: SVAWS, PMWI, TSS, and BCAP. Participants were compensated $\$ 40$ for completing the assessment.

\section{Results}

\section{Data cleaning}

There was no missing data for psychiatric diagnoses. There was minimal missing data for self-report questionnaires, ranging from $1.4-5.2 \%$ of the sample. Given the minimal amount of missing data, available item analysis was used (Parent, 2013). Skewness and kurtosis of the variables were within acceptable limits ( -2 to +2 : Tabachnick \& Fidell, 2007), with the exception of the current substance use diagnosis and childhood abuse perpetration by stranger variables. Because these variables were used as predictors rather than as criterion 
variables, we did not perform any variable transformations. Using the recommended cutoffs of the BCAP validity scales, $n=39$ cases were excluded as invalid. This resulted in a final sample of $n=211$ mothers.

\section{Descriptive statistics}

Of valid BCAP protocols $(n=211)$, participants had severe trauma histories. The majority of the sample witnessed IPV as a child (64.9\%), over a third experienced childhood physical abuse (37.9\%), and almost half experienced childhood sexual abuse (40.3\%). Of these childhood abuse victims, most reported experiencing childhood abuse multiple times, with 83.2\% experiencing more than one incident of physical abuse and $82.3 \%$ experiencing more than one incident of sexual abuse. Participants also had severe histories of IPV. The mean scores on the physical violence subscale of the SVAW were 15.00 (SD = 12.04), indicating participants frequently experienced trauma that included being kicked, stomped, or punched. Nearly half reported sexual abuse from the abuser who prompted them to seek shelter $(46.0 \%)$.

Many participants met criteria for IPV-related PTSD, 84.4\%. Depression was also common, $52.6 \%$. In addition, $4.3 \%$ met criteria for a substance use disorder, and $18.0 \%$ met criteria for borderline personality disorder. The average time in shelter at the time of the assessment was 22.1 days $(\mathrm{SD}=32.5)$.

\section{Aim 1 - Integrated Model of Child Abuse Potential}

We used a hierarchical multiple regression model computed with variables grouped in steps, following the developmental psychopathology framework. The first step was composed of childhood abuse variables, the second step current psychopathology, and the last step was variables related to recent IPV. Per recommendations in the literature (Haselschwerdt et al., 2017), we included variables designed to capture the heterogeneity of childhood abuse. Specifically, we used data from the TSS to create variables capturing distress, frequency, and relationship to the perpetrator of child maltreatment (e.g., family member, stranger, other; dummy coded). We also computed variables to reflect PTSD and depression symptom severity. For participants who did not meet criteria for PTSD or MDD, their scores were considered as zeros. Many participants were experiencing acute distress at the time of the diagnostic assessment and self-reported high symptoms but did not meet criteria for MDD or PTSD. Thus, we converted these scores to zero for those who did not meet criteria in order to prioritize examining the specific effects of these forms of psychopathology. We were unable to reflect severity in substance use or BPD diagnoses. Thus, our model incorporated nuanced and detailed variables for both childhood abuse and psychiatric diagnoses, better reflecting the lived experiences of our participants. Notably, in this analysis with 17 predictors (10 childhood abuse, 4 psychiatric, 3 IPV), only PTSD severity was a significant predictor of child abuse potential in the final step, and the overall model was not significant $\left(\mathrm{R}^{2}=.238\right.$, Power $\left.=.90\right)$.

We repeated this analysis with a simplified model to increase power (total predictors $=11$ ). Consistent with results from the first analysis, only PTSD severity and BPD diagnosis were significant predictors of child abuse potential in the final step. This model was significant, $F$ 
$(11,185)=4.35, p<.001$, with a medium-large effect size $\left(f^{2}=.26\right)$, Power $=1.00$, accounting for $20.6 \%$ of the variance); multicollinearity diagnostics were within limits, tolerance $>$.2. IPV variables did not improve prediction $\left(\mathrm{R}^{2}\right.$ change $=.01$, significant $F$ change $=.56)$. Results of this model are summarized in Table 1. Using a correction for multiple comparisons $(p<.01)$, only PTSD severity remains a significant predictor of child abuse potential.

\section{Aim 2 - Mediation Models}

Specifically, we were interested in whether the relationship between childhood histories of sexual abuse or physical abuse (coded dichotomously) and current CAP scores were mediated by IPV-related PTSD. Because distress from childhood abuse was significant in the regression model, we included this variable in preliminary mediation tests as well. Initial bivariate tests indicated that neither childhood physical abuse nor distress from physical abuse was correlated with PTSD scores; nor were childhood abuse variables (physical or sexual) correlated with a borderline personality disorder diagnosis.

Thus, we tested the relationship between distress from childhood sexual abuse, PTSD symptoms, and current CAP scores, using the PROCESS macro for SPSS (see Figure 1). There was a significant indirect effect of childhood sexual abuse on child abuse potential scores through PTSD symptoms, $a b=.08$, with a bootstrapped confidence interval [.03-.17] indicating significant mediation. Approximately $11 \%$ of the variance in child abuse potential was accounted for by the predictors. PTSD symptoms as a mediator of the relationship between distress from childhood sexual abuse and current child abuse potential accounted for approximately one-third of the total effect of the model, percent mediation $\left(P_{M}\right)=.32$.

\section{Aim 3 - Descriptive Data on BCAP Scores}

Scores ranged from 3 to 24 ( 24 being the highest possible score), median $=15.5$, mode $=$ 18.0. Utilizing the score of 12 and above as the cut-off for high risk, as suggested by Ondersma et al., (2005), 77.9\% of the sample would be considered high risk for child abuse potential. This is a much higher rate than most other research $(5.9 \%$ - Walker and Davies, $2012 ; 7.1-8.5 \%$ - Ondersma et al., 2005). Yet, this rate is similar to the $62.5 \%$ found by Rodriguez (2006), who utilized the full CAPI at a comparable cutoff point. Similarly, the mean total abuse risk score in our sample was 15.1 ( $\mathrm{SD}=4.5$ ), about twice the means found in other samples (Walker \& Davies, 2012; Ondersma et al., 2005). Table 2 summarizes how percent above the cut-off and mean BCAP scores compare across similar samples.

\section{Aim 4 - Exploration of BCAP Subscales}

We computed $t$-tests to compare BCAP subscale scores (distress, rigidity, and loneliness) for the three most common psychiatric diagnoses in our sample: current depression, PTSD, and BPD diagnoses (see Tables 3-5). We did not conduct these analyses for substance use diagnoses, given the very small number of substance use diagnoses in this sample (4.3\%). For MDD diagnoses, the distress and loneliness subscales were significantly higher for the depressed group. For PTSD diagnoses, all subscales were significantly higher for participants with PTSD diagnoses compared to those without PTSD. For BPD diagnoses, 
only the distress and loneliness subscales were significantly different. The rigidity subscale approached significance $(p=.06)$.

\section{Discussion}

Women in domestic violence shelters represent a unique high-risk sample for child abuse potential; they are women attempting to build safer, healthier lives while facing significant barriers. Results of the current study highlight the extreme violence experienced by shelter women and subsequent poor mental health; these findings are in line with previous research (e.g., Johnson, Zlotnick, \& Perez, 2008). Consistent with our hypotheses, we found that the racially diverse women in our sample had mean abuse risk scores much higher than those in prior research. Thus, women in shelter, while having minimized some risks in their lives by physically leaving abusive environments, remain a vulnerable population in terms of CAP.

In predicting CAP scores using a model that is accounting for multiple types of risk factors, we found that current psychopathology—in particular, IPV-related PTSD—was the most robust risk factor for CAP. Indeed, neither variables accounting for the wide range and context of child abuse experiences nor IPV variables were significant predictors. This was inconsistent with hypotheses that the recency of IPV exposure would be a significant risk factor (Rodriguez, 2006), but it is consistent with research in other samples demonstrating the importance of current psychopathology in predicting CAP (Rodriguez et al., 2016; Smith et al., 2014; Whiting et al., 2009). Indeed, Myers et al. (2015) demonstrated how it is the accumulation of traumatic and abusive experiences that is most related to mental health symptoms, rather than any one single experience. While it may be that the accumulation of adversity is most predictive of mental health problems, specific diagnoses are likely important for understanding CAP. Given the emotional reactivity and volatility of patients with PTSD and BPD, it is unsurprising that these disorders are associated with CAP (Rodriguez, Smith, \& Silvia, 2016). It is noteworthy that PTSD and BPD are some of the most common diagnoses associated with child abuse (e.g., Ackerman et al., 1998; Filipas \& Ullman, 2006; Rogosch \& Cicchetti, 2005).

We also conducted a mediation analysis to further specify the hypothesized pathway. Indeed, IPV-related PTSD symptoms were a significant mediator of the relationship between distress from childhood sexual abuse and current potential for engaging in childhood physical abuse. Unexpectedly, this relationship was specific to childhood sexual abuse and was not significant for childhood physical abuse. Although more research is needed to explore these relationships, there may be something especially damaging about sexual abuse above and beyond physical abuse, as it represents a more severe degree of psychological injury and/or violation to the victim. Distress from sexual abuse may be qualitatively different from distress from physical abuse, as it involves a different type of boundary violation and is more stigmatized (Kennedy \& Prock, 2016). This finding could also reflect that childhood sexual abuse frequently co-occurs with other types of controlling and manipulative behaviors on behalf of the perpetrator (Barnes, Noll, Putnam \& Trickett, 2009) that damage an individual's ability to form a healthy self-concept and regulate their emotions (Charak et al., 2017). 
Overall, these findings extend prior literature on the relationship of maternal history of childhood abuse to CAP (Bert et al., 2009; Milner et al., 2010) or history of childhood abuse to IPV (Jaffe et al., 2012) by using a developmental psychopathology framework to conceptualize a more complicated pathway. Indeed, we would suggest that this framework is necessary for our unique population: racially diverse women in shelter. Our findings highlight the importance of PTSD symptoms on child abuse potential, suggesting that attending to the current, specific mental health needs of women in the shelter is a very worthwhile intervention for both reducing the distress of mothers and potential harm to children.

Given the transience of women in domestic violence shelters and the limited resources available for these mothers and shelters (Ham-Rowbottom, Gordon, Jarvis \& Novaco, 2005), stepped care models are recommended. Stepped care models are service delivery approaches that address treatment barriers to help individuals in a more user-friendly and cost-effective way than standard treatment delivery systems (e.g., Salloum, Scheeringa, Cohen, \& Storch, 2014). Stepped care is particularly beneficial for a marginalized population such as women in shelter, who face barriers logistically (e.g., needing transportation and childcare) and personally (e.g., struggling with depression and/or PTSD that makes it difficult to get out of bed or leave the shelter). Stepped care delivery models typically begin with less therapist time and convenient interventions for patients, such as home-based care.

Within shelter, interventions that can be delivered by non-doctoral experts would have the greatest impact by being able to utilize existing resources (volunteers and bachelor's level staff). For example, mindfulness-based coping interventions such as mindful yoga could be beneficial (Allen \& Wozniak, 2011; Franzblau, Echevarria, Smith, \& Van Cantfort, 2008). Mindfulness-Based Stress Reduction (MBSR; Kabat-Zinn, 2003) has been used as a community-based intervention to treat low-income, racial minority women who have experienced IPV and a concomitant diagnosis of PTSD (Dutton, Bermudez, Matas, Majid, \& Myers, 2013). MBSR does not require a clinician for effective delivery and can be delivered in a group format, effectively reducing the stigma surrounding its use and making it more accessible.

We highly recommend that providers be aware of the additional barriers to accessing care faced by this population, including poverty and the need for childcare (Ham-Rowbottom, Gordon, Jarvis \& Novaco, 2005). Thus, referrals to providers that provide childcare, offer sliding scale fees, and offer services outside of traditional office hours are recommended. The multiple barriers faced by this population highlights the importance of systemic issues in healthcare and their relation to the oppression of women and children. Few healthcare settings offer childcare or non-traditional hours for services, yet these are substantial barriers for some of the most vulnerable people in our society. We highly recommend traumainformed approaches across all levels of care (Johnson, Zlotnick, \& Perez, 2011). Further, given that most of the sample was considered high risk for child abuse potential, it is likely that at least some participants had already abused their children. Thus, trauma-informed approaches and stepped care models that can be utilized by the entire family are critical. It is likely that many of the children who seek shelter with their mothers also suffer from PTSD. 
Finally, we conducted exploratory analyses to examine the potential usefulness of the BCAP subscales in further understanding the relationship between psychiatric diagnoses and CAP. Notably, most of the suggested research subscales were not sufficiently internally consistent for use in this study, suggesting that the factor structure proposed by Ondersma et al. (2005) may not apply to racially diverse mothers in shelter. Some of the subscales that were most unreliable were also constructs that would be expected to be disrupted among families in shelter, such as family conflict, persecution, happiness, and financial stress. This is not to suggest that the BCAP total abuse score is lacking validity; Ondersma et al. (2005) introduced the subscales for research purposes, while the total abuse risk score is recommended for clinical evaluations.

We found that for all three diagnoses (depression, PTSD, and BPD), the distress and loneliness subscales significantly differed between the diagnostic groups. This is consistent with Dawe et al. (2017) who found the loneliness subscale accounted for most of the variance in BCAP scores in mothers in opioid-substitution therapy. This provides further support for the necessity of interventions that can reduce psychiatric distress in shelter and indicates that perhaps loneliness is a unique risk factor for child abuse potential in women in shelter. It may be that experiences of violence perpetrated by intimates - including child abuse and IPV (Kulkarni, 2009; Rokach, 2007) - are particularly related to feelings of loneliness and the consequent risk for child abuse (Gaudin, Polansky, Kilpatrick, \& Shilton, 1995). Thus, in addition to trauma-focused interventions to decrease psychopathology, interventions and policies that facilitate positive social support for these women may also decrease CAP.

\section{Limitations}

Foremost, IPV survivors living in shelters are a marginalized group with unique vulnerabilities, and findings cannot be generalized to all IPV survivors. Because all the women in the sample experienced sufficiently severe IPV for women to seek shelter, any relationships between IPV and the other factors may be attenuated, as there are no women without these severe victimization experiences in the sample. Generally, the retrospective nature of this research can create a risk of bias in the memory of survivors. It is likely that not all cases of prior childhood physical and sexual abuse were effectively captured by the TSS; we recommend future research use more comprehensive measures that also include childhood neglect.

Additionally, DSM-IV symptom profiles for PTSD, BPD, and MDD were used in the current study, which may differ from the new classifications in DSM-5. Furthermore, PTSD was assessed only as related to the IPV, even though we hypothesized that PTSD related to childhood abuse would be related to risk of abusing. While we utilized the widely used and well-validated BCAP (Ondersma et al., 2005) to assess CAP, a more comprehensive measurement of CAP is recommended for future research. We suggest future research integrate behavioral analogs of CAP (in line with Rodriguez et al., 2016), in addition to selfreports. Finally, this study utilized a cross-sectional design, but true mediation testing requires longitudinal data. Longitudinal research that can account for the timing of 
childhood abuse, IPV, and subsequent psychopathology is recommended and would be a helpful application of the developmental psychopathology framework.

\section{Conclusions}

The greatest predictor of CAP among the marginalized population of women domestic violence shelter residents is the presence of IPV-related PTSD. Our findings indicate that somewhat contrary to prior research, histories of trauma were not the most powerful predictors for CAP among women in shelter. Rather, the psychological disorders that result from exposure to trauma mediated these effects. This is a hopeful finding; although providers cannot change an individual's past, they can effectively treat PTSD and thus reduce the risk of child abuse. In sum, serving the mental health needs of women in shelter not only improves the health and well-being of the women who have experienced IPV but the health and well-being of their children. By effectively treating PTSD, medical providers can help prevent the cycle of child abuse from continuing into the next generation.

\section{Acknowledgments:}

We are grateful to the large number of graduate and undergraduate HOPE team members and numerous shelter staff for their assistance and dedication to this project. We also appreciate each and every participant who shared their experiences with us.

Funding: This work was supported by grant \#MH095767 awarded to Dr. Johnson

\section{References}

Ackerman PT, Newton JE, McPherson WB, Jones JG, \& Dykman RA (1998). Prevalence of posttraumatic stress disorder and other psychiatric diagnoses in three groups of abused children (sexual, physical, and both). Child Abuse \& Neglect, 22, 759-774. [PubMed: 9717613]

Allen KN, \& Wozniak DF (2011). The language of healing: Women's voices in healing and recovering from domestic violence. Social Work in Mental Health, 9, 37-55.

Banyard VL (2013). Go big or go home: Reaching for a more integrated view of violence prevention. Psychology of Violence, 3, 115-120. 10.1037/a0032289

Barnes JE, Noll JG, Putnam FW, \& Trickett PK (2009). Sexual and physical revictimization among victims of severe childhood sexual abuse. Child Abuse \& Neglect, 33(7), 412420 10.1016/j.chiabu. 2008.09.013

Berg-Nielsen TS, Vikan A, \& Dahl AA (2002). Parenting related to child and parental psychopathology: A descriptive review of the literature. Clinical Child Psychology \& Psychiatry, 7 , 529-552. 10.1177/1359104502007004006

Bert SC, Guner BM, \& Lanzi RG (2009). The influence of maternal history of abuse on parenting knowledge and behavior. Family Relations, 58, 176-187. [PubMed: 21695065]

Blake DD, Weathers FW, Nagy LM, Kaloupek DG, Gusman FD, Charney DS, \& Keane TM (1995). The development of a Clinician-Administered PTSD Scale. Journal of Traumatic Stress, 8, 75-90. 10.1002/jts.2490080106 [PubMed: 7712061]

Charak R, DiLillo D, Messman-Moore TL, \& Gratz KL (in press) Latent classes of lifetime sexual victimization characteristics in women in emerging adulthood: Differential relations with emotion dysregulation. Psychology of Violence. doi:10.1037/vio0000154

Cicchetti D, \& Toth SL (2009). The past achievements and future promises of developmental psychopathology: The coming of age of a discipline. Journal of Child Psychology and Psychiatry, 50, 16-25. 10.1111/j.1469-7610.2008.01979.x [PubMed: 19175810] 
Dawe S, Taplin S, \& Mattick RP (2017). Psychometric investigation of the Brief Child Abuse Potential Inventory in mothers on opioid substitution therapy. Journal of Family Violence, 32, 341-348. doi: 10.1007/s10896-016-9821-3

Dutton MA, Bermudez D, Matas A, Majid H, \& Myers NL (2013). Mindfulness-based stress reduction for low-income, predominantly African American women with PTSD and a history of intimate partner violence. Cognitive \& Behavioral Practice, 20, 23-32. [PubMed: 24043922]

Eaton WW, Smith C, Ybarra M, Muntaner C, \& Tien A (2004). Center for Epidemiologic Studies Depression Scale: Review and Revision (CESD and CESD-R) In The use of psychological testing for treatment planning and outcomes assessment: Volume 3: Instruments for adults (3rd ed) (pp. 363-377). Mahwah, NJ: Lawrence Erlbaum Associates Publishers.

Filipas HH, \& Ullman SE (2006). Child sexual abuse, coping responses, self-blame, posttraumatic stress disorder, and adult sexual revictimization. Journal of Interpersonal Violence, 21, 652-672. [PubMed: 16574638]

First MB, Spitzer RL, Gibbon M, \& Williams JBW (2002). Structured Clinical Interview for DSM-IVTR Axis 1 Disorders, Research Version, Patient Edition with Psychotic Screen (SCID-I/P W/PSY SCREEN). New York, NY, US.

Franzblau SH, Echevarria S, Smith M, \& Van Cantfort TE (2008). A preliminary investigation of the effects of giving testimony and learning yogic breathing techniques on battered women's feelings of depression. Journal of Interpersonal Violence, 23, 1800-1808. [PubMed: 18319369]

Gaudin JM, Polansky NA, Kilpatrick AC, \& Shilton P (1993). Loneliness, depression, stress, and social supports in neglectful families. The American Journal of Orthopsychiatry, 63, 597-605. 10.1037/h0079475 [PubMed: 8267100]

Gobin RL, Iverson KM, Mitchell K, Vaughn R, \& Resick PA (2013). The impact of childhood maltreatment on PTSD symptoms among female survivors of intimate partner violence. Violence \& Victims, 28, 984-999. doi:10.1891/0886-6708.VV-D-12-00090 [PubMed: 24547676]

Grady MA, \& Bloom KC (2004). Pregnancy outcomes of adolescents enrolled in a centering pregnancy program. Journal of Midwifery \& Women's Health, 49, 412-420.

Hamby S, \& Grych J (2013). Tracing the threads of the web: The epidemiology of interconnections among forms of violence and victimization In The Web of Violence (pp. 8-27). New York, NY, US: Springer 10.1007/978-94-007-5596-3_2

Ham-Rowbottom KA, Gordon EE, Jarvis KL, \& Novaco RW (2005). Life constraints and psychological well-being of domestic violence shelter graduates. Journal of Family Violence, 20, 109-121. 10.1007/s 10896-005-3174-7

Haselschwerdt ML, Savasuk-Luxton R, \& Hlavaty K (2017). A methodological review and critique of the "Intergenerational Transmission of Violence" literature. Trauma, Violence, \& Abuse, $10.1177 / 1524838017692385$

Hazen AL, Connelly CD, Roesch SC, Hough RL, \& Landsverk JA (2009). Child maltreatment profiles and adjustment problems in high-risk adolescents. Journal of Interpersonal Violence, 24, 361-378. 10.1177/0886260508316476 [PubMed: 18391059]

Iverson KM, Litwack SD, Pineles SL, Suvak MK, Vaughn RA, \& Resick PA (2013). Predictors of intimate partner violence revictimization: The relative impact of distinct PTSD symptoms, dissociation, and coping strategies. Journal of Traumatic Stress, 26, 102-110. 10.1002/jts.21781 [PubMed: 23417878]

Jaffe AE, Cranston CC, \& Shadlow JO (2012). Parenting in females exposed to intimate partner violence and childhood sexual abuse. Journal of Child Sexual Abuse: Research, Treatment, \& Program Innovations for Victims, Survivors, \& Offenders, 21, 684-700.

Johnson DM, Zlotnick C, \& Perez S (2008). The relative contribution of abuse severity and PTSD severity on the psychiatric and social morbidity of battered women in shelters. Behavior Therapy, 39, 232-241. 10.1016/j.beth.2007.08.003 [PubMed: 18721637]

Johnson DM, Zlotnick C, \& Perez S (2011). Cognitive behavioral treatment of PTSD in residents of battered women's shelters: Results of a randomized clinical trial. Journal of Consulting \& Clinical Psychology, 79, 542-551. 10.1037/a0023822 [PubMed: 21787052]

Jordan CE (2009). Advancing the study of Violence Against Women. Violence Against Women, 15, 393-419. 10.1177/1077801208330692 [PubMed: 19176314] 
Juby C, Downs W, \& Rindels B (2014). Intimate partner violence victimization, maternal child maltreatment, and the mediating impact of changes in family structure. Child \& Adolescent Social Work Journal, 31, 237-249. doi:10.1007/s10560-013-0318-0

Kabat-Zinn J (2003). Mindfulness-based interventions in context: Past, present, and future. Clinical Psychology: Science \& Practice, 10, 144-156.

Kelley ML, Lawrence HR, Milletich RJ, Hollis BF, \& Henson JM (2015). Modeling risk for child abuse and harsh parenting in families with depressed and substance-abusing parents. Child Abuse \& Neglect, 43, 42-52. 10.1016/j.chiabu.2015.01.017 [PubMed: 25724658]

Kennedy AC, \& Prock KA (2016). "I Still Feel Like I Am Not Normal" A Review of the Role of Stigma and Stigmatization Among Female Survivors of Child Sexual Abuse, Sexual Assault, and Intimate Partner Violence. Trauma, Violence, \& Abuse, 1524838016673601.

Kulkarni SJ (2009). The relational consequences of interpersonal violence (IPV) for adolescent mothers. Youth \& Society, 41, 100-123. 10.1177/0044118X08318120

Lannert BK, Levendosky AA, \& Bogat GA (2013). The interaction of maternal personality traits and intimate partner violence as influences on maternal representations. Infant Mental Health Journal, 34, 222-233. doi:10.1002/imhj.21385

Laulik S, Chou S, Browne KD, \& Allam J (2013). The link between personality disorder and parenting behaviors: A systematic review. Aggression and Violent Behavior, 18, 644-655. 10.1016/j.avb. 2013.07.017

Lobbestael J, Leurgans M, \& Arntz A (2011). Inter-rater reliability of the Structured Clinical Interview for DSM-IV Axis I Disorders (SCID I) and Axis II Disorders (SCID II). Clinical Psychology \& Psychotherapy, 18, 75-79. 10.1002/cpp.693 [PubMed: 20309842]

MacMillan HL, \& Wathen CN (2005). Family violence research: Lessons learned and where from here? JAMA, 294, 618 10.1001/jama.294.5.618 [PubMed: 16077058]

Marshall LL (1992). Development of the Severity of Violence Against Women Scales. Journal of Family Violence, 7, 103-121. 10.1007/BF00978700

Milner JS, \& Wimberley RC (1979). An inventory for the identification of child abusers. Journal of Clinical Psychology, 35, 95-100. 10.1002/1097-4679(197901)35:1 [PubMed: 422737]

Milner JS, Thomsen CJ, Crouch JL, Rabenhorst MM, Martens PM, Dyslin CW, \& ... Merrill LL (2010). Do trauma symptoms mediate the relationship between childhood physical abuse and adult child abuse risk? Child Abuse \& Neglect, 34, 332-344. doi:10.1016/j.chiabu.2009.09.017 [PubMed: 20359748]

Myers HF, Wyatt GE, Ullman JB, Loeb TB, Chin D, Prause N, ... Liu H (2015). Cumulative burden of lifetime adversities: Trauma and mental health in low-SES African Americans and Latino/as. Psychological Trauma: Theory, Research, Practice, and Policy, 7, 243-251. 10.1037/a0039077

Noll JG, Trickett PK, Harris WW, \& Putnam FW (2008). The cumulative burden borne by offspring whose mothers were sexually abused as children: Descriptive results from a multigenerational study. Journal of Interpersonal Violence, 24, 424449.

Norris FH (1992). Epidemiology of trauma: Frequency and impact of different potentially traumatic events on different demographic groups. Journal of Consulting \& Clinical Psychology, 60, 409418. 10.1037/0022-006X.60.3.409 [PubMed: 1619095]

Norris FH, \& Perilla JL (1996). The revised civilian Mississippi scale for PTSD: Reliability, validity, and cross-language stability. Journal of Traumatic Stress, 9, 285-298. [PubMed: 8731548]

Ondersma SJ, Chaffin MJ, Mullins SM, \& LeBreton JM (2005). A Brief Form of the Child Abuse Potential Inventory: Development and Validation. Journal of Clinical Child \& Adolescent Psychology, 34, 301-311. doi:10.1207/s15374424jccp3402_9 [PubMed: 15901230]

Parent MC (2013). Handling Item-Level Missing Data. The Counseling Psychologist, 41, 568-600. $10.1177 / 0011000012445176$

Rodriguez CM (2006). Emotional functioning, attachment style, and attributions as predictors of child abuse potential in domestic violence victims. Violence \& Victims, 21, 199-212. 10.1891/vivi. 21.2.199 [PubMed: 16642739]

Rodriguez CM, Smith TL, \& Silvia PJ (2016). Parent-child aggression risk in expectant mothers and fathers: A multimethod theoretical approach. Journal of Child and Family Studies, 25, 3220-3235. 10.1007/s10826-016-0481-y [PubMed: 28082826] 
Rogosch FA, \& Cicchetti D (2005). Child maltreatment, attention networks, and potential precursors to borderline personality disorder. Development \& Psychopathology, 17, 1071-1089. [PubMed: 16613431]

Rokach A (2007). Loneliness and intimate partner violence. Social Work in Health Care, 45, 19-31. 10.1300/J010v45n01_02

Salloum A, Scheeringa MS, Cohen JA, \& Storch EA (2014). Development of stepped care traumafocused cognitive-behavioral therapy for young children. Cognitive \& Behavioral Practice, 21, 97108. [PubMed: 25411544]

Schneider R, Baumrind N, \& Kimerling R (2007). Exposure to child abuse and risk for mental health problems in women. Violence and Victims, 22, 620. [PubMed: 18064973]

Shenk CE, Ammerman RT, Teeters AR, Bensman HE, Allen EK, Putnam FW, \& Van Ginkel JB (2017). History of maltreatment in childhood and subsequent parenting stress in at-risk, first-time mothers: Identifying points of intervention during home visiting. Prevention Science, 18, 361-370. 10.1007/s11121-017-0758-4 [PubMed: 28168607]

Smith AL, Cross D, Winkler J, Jovanovic T, \& Bradley B (2014). Emotional dysregulation and negative affect mediate the relationship between maternal history of child maltreatment and maternal child abuse potential. Journal of Family Violence, 29, 483-494. doi:10.1007/ s10896-014-9606-5

Smith DK, Leve LD, \& Chamberlain P (2006). Adolescent girls' offending and health-risking sexual behavior: The predictive role of trauma. Child Maltreatment, 11, 346-353. [PubMed: 17043319]

Tabachnick BG, \& Fidell LS (2007). Using multivariate statistics, 5th ed. Boston, MA: Allyn \& Bacon/Pearson Education.

Tolman RM (1999). The validation of the Psychological Maltreatment of Women Inventory. Violence \& Victims, 14, 25-37. [PubMed: 10397624]

Trimble DD, Nava A, \& McFarlane J (2013). Intimate partner violence and antiretroviral adherence among women receiving care in an urban southeastern Texas HIVclinic. JANAC: Journal of the Association of Nurses in AIDS Care, 24, 331-340. [PubMed: 23790276]

U.S. Department of Health and Human Services, Administration for Children and Families, Administration on Children, Youth and Families, Children's Bureau. (2012) Child Maltreatment 2012 [online]. Washington, DC: Government Printing Office; 2012.

Walker CA, \& Davies J (2010). A critical review of the psychometric evidence base of the Child Abuse Potential Inventory. Journal of Family Violence, 25, 215-227.

Walker CA, \& Davies J (2012). A cross-cultural validation of the Brief Child Abuse Potential Inventory (BCAP). Journal of Family Violence, 27, 697-705.

Weiss DS (2004). Structured Clinical Interview Techniques for PTSD In Wilson JP \& Keane TM (Eds.), Assessing psychological trauma and PTSD (2nd ed., pp. 103-121). The Guilford Press.

Whiting JB, Simmons LA, Havens JR, Smith DB, \& Oka M (2009). Intergenerational transmission of violence: The Influence of self-appraisals, mental disorders and substance abuse. Journal of Family Violence, 24, 639-648. 10.1007/s10896-009-9262-3

Widom CS, \& Wilson HW (2015). Intergenerational transmission of violence In Violence and Mental Health (pp. 27-45). Dordrecht: Springer Netherlands 10.1007/978-94-017-8999-8_2 


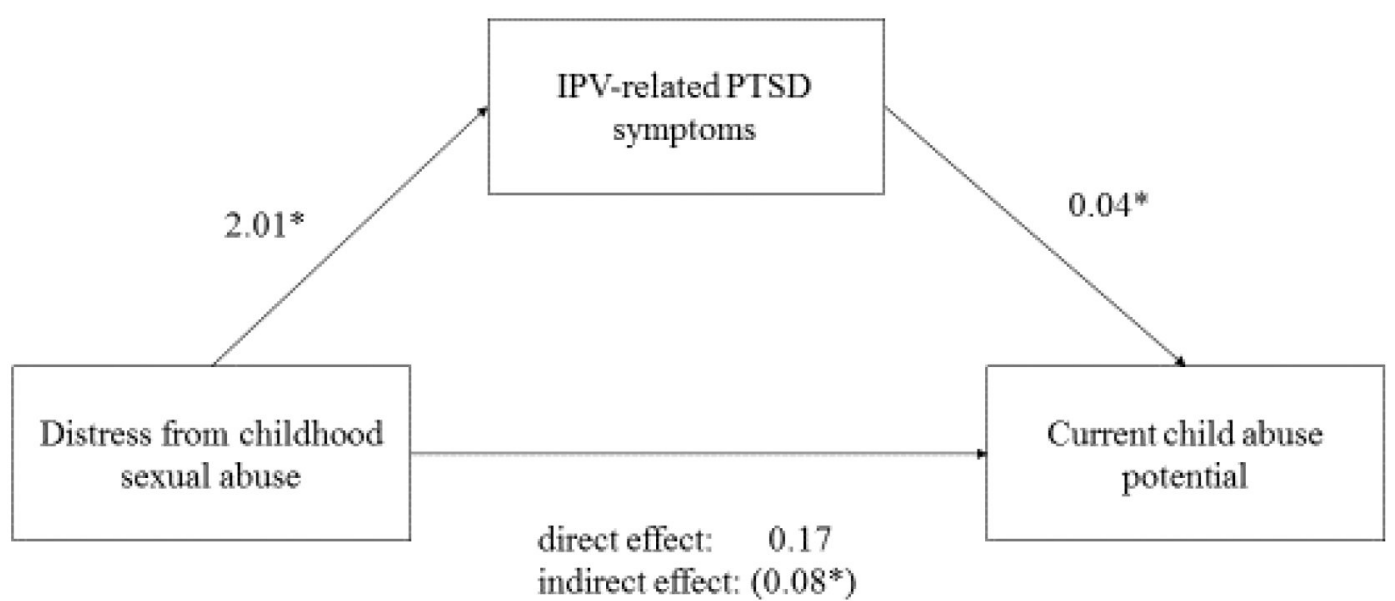

Figure 1. Mediation Model with Coefficients

Note. * indicates $p<.01$ 
Table 1

Hierarchical Regression Analysis Summaries for Predicting Child Abuse Potential in Final Model

\begin{tabular}{|c|c|c|c|c|c|c|}
\hline Step and Predictor Variable & $\boldsymbol{B}$ & $S E B$ & & $R^{2}$ & $\Delta R^{2}$ & $p$ \\
\hline Step 1: childhood abuse & & & & .07 & .07 & $.006^{*}$ \\
\hline physical abuse distress & .29 & .15 & .19 & & & $.05^{*}$ \\
\hline family member perpetrator (physical) & .33 & 1.20 & .03 & & & .79 \\
\hline sexual abuse distress & .11 & .12 & .08 & & & .35 \\
\hline family member perpetrator (sexual) & .41 & 1.25 & .03 & & & .75 \\
\hline Step 2: psychiatric diagnoses & & & & .20 & .12 & $<.001 *$ \\
\hline physical abuse distress & .23 & .14 & .15 & & & .11 \\
\hline family member perpetrator (physical) & .40 & 1.13 & .04 & & & .73 \\
\hline sexual abuse distress & -.01 & .11 & -.01 & & & .92 \\
\hline family member perpetrator (sexual) & .96 & 1.19 & .07 & & & .42 \\
\hline PTSD severity & .04 & .01 & .27 & & & $<.001 *$ \\
\hline Depression severity & .02 & .02 & .07 & & & .37 \\
\hline SUD (yes/no) & 2.67 & 1.49 & .12 & & & .08 \\
\hline borderline personality (yes/no) & 1.68 & .80 & .14 & & & $.04^{*}$ \\
\hline Step 3: IPV victimization & & & & .21 & .01 & $<.001^{*}$ \\
\hline physical abuse distress & .24 & .14 & .16 & & & .09 \\
\hline family member perpetrator (physical) & .33 & 1.14 & .03 & & & .77 \\
\hline sexual abuse distress & -.01 & .11 & -.01 & & & .92 \\
\hline family member perpetrator (sexual) & .82 & 1.20 & .06 & & & .50 \\
\hline PTSD severity & .04 & .01 & .27 & & & $.001^{*}$ \\
\hline Depression severity & .02 & .02 & .07 & & & .37 \\
\hline SUD (yes/no) & 2.76 & 1.50 & .12 & & & .07 \\
\hline borderline personality (yes/no) & 1.58 & .81 & .14 & & & $.05^{*}$ \\
\hline severity psychological IPV & .78 & .70 & .09 & & & .27 \\
\hline severity physical and sexual IPV & -.48 & .37 & -.10 & & & .20 \\
\hline time since contact with abuser & .09 & .31 & .02 & & & .77 \\
\hline
\end{tabular}

Note. $\mathrm{PTSD}=$ posttraumatic stress disorder, SUD = substance use disorder

indicates statistically significant difference 


\section{Table 2}

Comparison of mean abuse risk scale scores across studies

\begin{tabular}{|l|l|l|l|}
\hline Authors & Sample Description & $\boldsymbol{M}, \boldsymbol{S D}$ & $\begin{array}{l}\text { \% at or above } \\
\text { cutof }\end{array}$ \\
\hline Ondersma et al., 2005 & $N=1470$, Parents enrolled in child abuse prevention and/or treatment services & $7.1,6.0$ & $\mathrm{n} / \mathrm{a}$ \\
\hline Ondersma et al., 2005 & $N=713$, Parents enrolled in child abuse prevention and/or treatment services & $8.5,6.3$ & $\mathrm{n} / \mathrm{a}$ \\
\hline Rodriguez 2006 & $N=39$, Women with IPV histories, average of 17 days away from abusers & $\mathrm{n} / \mathrm{a}$ & $62.5^{\dagger}$ \\
\hline Rodriguez 2006 & $N=41$, Women with IPV histories, average of 10 months away from abusers & $\mathrm{n} / \mathrm{a}$ & $62.5^{\dagger}$ \\
\hline Walker \& Davies, 2012 & $N=324$, Parents in large city in United Kingdom & $5.89,3.60$ & 7.7 \\
\hline Current Study & $N=208$, Women residing in IPV shelters, average 22 days away from abusers & $15.1,4.5$ & 77.8 \\
\hline
\end{tabular}

Note. IPV = intimate partner violence,

${ }^{\dagger} \%$ cut-off was reported for $\mathrm{N}=80$, combining the two sub-samples 
Table 3

T-test results for child abuse potential subscales by depression diagnosis

\begin{tabular}{|l|l|l|l|l|l|l|}
\hline & MDD Yes, $\boldsymbol{n}=\mathbf{1 0 7}$ & $\underline{\text { MDD no, } \boldsymbol{n = 6 1}}$ & & & & \\
\hline BCAP subscale & $\mathbf{M}, \boldsymbol{S D}$ & $\boldsymbol{M}, \boldsymbol{S D}$ & df & $\boldsymbol{t}$ & $\boldsymbol{p}$ & Cohen's $\boldsymbol{d}$ \\
\hline distress & $4.17,1.90$ & $2.66,2.02$ & 119.0 & 4.77 & $<.001^{*}$ & 0.87 \\
\hline rigid & $2.38,1.17$ & $2.39,1.39$ & 105.1 & .03 & .98 & 0.08 \\
\hline loneliness & $3.43,1.07$ & $2.33,1.70$ & 87.4 & 4.58 & $<.001^{*}$ & 0.83 \\
\hline total & $15.79,4.21$ & $13.87,4.77$ & 167 & 2.70 & $<.01^{*}$ & 0.73 \\
\hline
\end{tabular}

Note. MDD - Major Depressive Disorder. BCAP = Brief Child Abuse Potential Inventory indicates statistically significant difference 


\section{Table 4}

T-test results for child abuse potential subscales by Posttraumatic stress disorder (PTSD) diagnosis

\begin{tabular}{|l|l|l|l|l|l|l|}
\hline & PTSD Yes, $\boldsymbol{n}=\mathbf{1 7 8}$ & PTSD no, $\boldsymbol{n}=\mathbf{3 3}$ & & & & \\
\hline BCAP subscale & $\boldsymbol{M}, \boldsymbol{S D}$ & $\boldsymbol{M}, \boldsymbol{S D}$ & $\mathbf{d f}$ & $\boldsymbol{t}$ & $\boldsymbol{p}$ & Cohen's $\boldsymbol{d}$ \\
\hline distress & $3.78,1.99$ & $1.85,1.87$ & 205 & 5.15 & $<.001^{*}$ & 0.98 \\
\hline rigid & $2.51,1.19$ & $2.00,1.34$ & 203 & 2.17 & $<.05^{*}$ & 0.42 \\
\hline loneliness & $3.28,1.23$ & $1.94,1.77$ & 38 & 4.19 & $<.001^{*}$ & 1.01 \\
\hline total & $15.79,4.17$ & $11.61,4.61$ & 42.4 & 4.85 & $<.000^{*}$ & 0.99 \\
\hline
\end{tabular}

Note. $\mathrm{BCAP}=$ Brief Child Abuse Potential Inventory,

indicates statistically significant difference 
Table 5

T-test results for child abuse potential subscales by Borderline personality disorder (BPD) diagnosis

\begin{tabular}{|l|l|l|l|l|l|l|}
\hline & BPD Yes, $\boldsymbol{n}=\mathbf{3 7}$ & BPD no, $\boldsymbol{n}=\mathbf{1 7 2}$ & & & & \\
\hline BCAP subscale & $\boldsymbol{M}, \boldsymbol{S D}$ & $\boldsymbol{M}, \boldsymbol{S D}$ & df & $\boldsymbol{t}$ & $\boldsymbol{p}$ & Cohen's $\boldsymbol{d}$ \\
\hline distress & $4.75,1.63$ & $3.20,2.09$ & 61.9 & 4.91 & $<.001^{*}$ & 0.77 \\
\hline rigid & $2.78,1.12$ & $2.36,1.24$ & 203 & 1.89 & .06 & 0.34 \\
\hline loneliness & $3.70,0.81$ & $2.94,1.48$ & 95.5 & 4.39 & $<.001^{*}$ & 0.55 \\
\hline total & $17.26,3.26$ & $14.67,4.60$ & 70.9 & 4.04 & $<.001^{*}$ & 0.59 \\
\hline
\end{tabular}

Note. BCAP $=$ Brief Child Abuse Potential Inventory,

indicates statistically significant difference 